\title{
ON LAGRANGIAN MANIFOLD ADMITTING F (7, 1)-STRUCTURE
}

\author{
Pravin Kumar Srivastava ${ }^{1}$, Praveen Kumar Mathur ${ }^{2}$ \\ 1Department of Applied Science \& Humanities, Bundelkhand Institute of Engineering \& Technology, JHANSI \\ 2Department of Mathematics, Surya School of Planning \& Engineering College, LUCKNOW
}

\section{INTRODUCTION}

Lagrangian manifold admitting $\mathrm{F}(3,1)$-structure defined and studied by J. Nikie (1). Das Lovejoy and Nivas Ram (2) defined and studied Lagrangian manifold admittind $\mathrm{F}_{\lambda}$ polynomial structure and obtained equivalent conditions for vertical subspace $T_{v}(E)$, horizontal subspace $T_{H}(E)$ and enveloping space $T(E)$ in a Lagrangian manifold. In the present paper, I have proved equivalent conditions of vertical subspace $T_{V}(E)$, horizontal subspace $T_{H}(E)$ and the space $T(E)$ for Lagrangian manifold admitting $F(7,1)$-structure. Some other results concerning orthogonality of distributions have also been obtained.

Stein manifolds are complex manifolds $M$ which can be properly holomor-phically embedded into $C^{\mathbf{n}}$. They have a natural symplectic form obtained from $\mathrm{C}^{\mathrm{n}}$ which is a biholomorphic invariant. The richness of the interplay between Stein geometry and symplectic geometry was originally pointed out by Eliashberg and Gromov ([4], [5], [6]).

My research takes motivation from the following problems:

(1) Smooth affine varieties: What does the natural symplectic structure on the smooth affine variety say about its algebraic properties and vice versa?

(2) Uniqueness problems: Given a manifold $\mathrm{M}$, how many Stein/affine structures can we put on $M$ up to symplectomorphism? What can we say about contact structures?

(3) Dynamical questions: What are the dynamical properties of sym- plectic/contact manifolds coming from Stein geometry?

(4) Quantative questions: Quantative questions are ones such as: what are the largest symplectic objects that can be embedded in other symplectic objects? So in general we could ask what quantative symplectic properties can we obtain from Stein/affine manifolds?

Smooth affine varieties are an interesting subfamily of Stein manifolds. Some interesting questions in this field are:

(1) Which symplectic manifolds are symplectomorphic to smooth affine varieties?
(2) What is the relationship between the algebraic structure and the symplectic structure of an affine variety?

(3) Can we calculate symplectic homology of smooth affine varieties?

This question (and the next one) is interesting as it is related to a conjecture called the homological mirror symmetry conjecture.

(4) For certain pairs of Lagrangians can we calculate their wrapped Floer homology group? 1

One important family of symplectic manfiolds are cotangent bundles of smooth manifolds. The cotangent bundle of the sphere is symplectomorphic to the smooth affine variety $\left\{\mathbf{P}_{\mathbf{i}} \mathrm{z}^{2}=1\right\}$. We say that a simply connected compact manifold $\mathrm{Q}$ is has large free loopspace growth if the Betti numbers of its free loopspace grow faster than any polynomial of degree dimRQ.

Theorem 1 [19] The cotangent bundle of a manifold with large free loopspace growth is not symplectomorphic to any smooth affine variety.

In fact we can prove a stronger theorem where (roughly) we can allow the symplectomorphism to be ill defined inside

some compact subset of $\mathrm{T}^{*} \mathrm{Q}$.

There are many examples of manifolds of large free loopspace growth such as the connect sum of two simply connected manifolds where both manifolds have cohomology algebras with at least two generators. We prove this the- orem by using an invariant called the growth rate of symplectic homology. We show that the growth rate of these cotangent bundles is infinite whereas the growth rate of smooth affine varieties is finite.

The above theorem can be generalized as follows: We say that a simply connected manifold $Q$ has large based loopspace growth if the Betti numbers of its based loopspace grow faster than any polynomial of degree $\operatorname{dim} R Q$.

Theorem 2 The cotangent bundle of a manifold with large based loopspace growth is not symplectomorphic to any 
smooth affine variety. For instance this is true if the manifold is simply connected and its Betti numbers are sufficently large.

If we have two possibly non-compact Lagrangians inside some Stein man- ifold which behave in a nice way at infinity then we can assign to them a value in $\{-\infty\} \cup[0, \infty]$ called the growth rate of wrapped Floer cohomology.

The above theorem follows from the following claim combined with work in [1]:

Claim 1 Let $\mathrm{X}$ be a smooth affine variety and $\mathrm{S}$ a Stein manifold sym- plectomorphic to $X$. Then the growth rate of wrapped Floer cohomology for any two Lagrangians in $\mathrm{S}$ is $\leq \operatorname{dim}_{\mathrm{C}} \mathrm{X}$.

The methods used in Theorem 1 and Claim 3 combined with a compact- ness result should be able to help us compute symplectic homology as an algebra for a large family of smooth affine varieties. Symplectic homology has other product structures and for some specific examples of smooth affine varieties we should be able to calculate these structures. For instance a nice compactification $\mathrm{X}$ of a smooth affine variety $\mathrm{X}$ has a natural stratifica- tion and if the dimension $\mathrm{d}$ part of this stratification does not have a $\mathrm{C}$ passing through every point for all $\mathrm{d}>0$ then we should be able to show that symplectic homology is some deformation of an explicit graded algebra. This deformation

should controlled by maps from $\mathrm{C}^{*}$ into $\mathrm{X}$ although understanding this completely might be a difficult task. One good family of examples comes from [10]. Calculating what symplectic homology is would be very useful in helping us to understand a conjecture called the homo- logical mirror symmetry conjecture. One could also look at wrapped Floer cohomology of Lagrangians inside these manifolds again by using methods from the proof of Claim 1. The first Lagrangians I am hoping to look at are ones which intersect components of the stratification of $\mathrm{X}$ in a nice way.

One way of studying algebraic varieties is to look at the numerical prop- erties of the canonical class of our variety. For many years this has been a very effective way to study varieties. One can look at this set of ideas from a topological or symplectic perspective. People have done this a lot for projective varieties (See for instance [7] and [20]) but very little has been done for open varieties. One important invariant of affine varieties coming from these ideas is called log Kodaira dimension. For instance we have the following theorem:

Theorem 3 The $\log$ Kodaira dimension of a smooth affine variety A of complex dimension 2 whose homology is concentrated in degree 0 is a sym- plectic invariant. We also have partial invariance results in complex dimen- sion 3.

We prove this by embedding our affine variety as an open subset of a projective variety and then by looking at Gromov Witten invariants of this projective variety. We also hope to prove the following claim:

Claim 2 Suppose A is an affine variety such that it can be embedded as an open subset of a projective variety $\mathrm{X}$ such that the anticanonical class of $\mathrm{X}$ is homologous to an effective divisor in $\mathrm{X}$ disjoint from $\mathrm{A}$. Then any affine variety symplectomorphic to A has log Kodaira dimension less than 1 .

To prove this we show that the symplectic homology of A is zero in nega- tive degrees but we can show that symplectic homology of any affine variety with positive log Kodaira dimension is non-zero in some negative degree. The methods used to prove the above claim should have other applications.

For instance if an affine variety $A$ is equal to $X \backslash D$ where (1) $X$ is projective.

(2) $\mathrm{D}$ is a smooth normal crossing divisor. (3) $\mathrm{KX}+\mathrm{D}$ is ample

then one should be able to compute symplectic homology. In fact symplectic homology might even be able to extract information such as the number of components of $\mathrm{D}$.

Another effective way to study algebraic varieties is to look at rational curves on them and again we can look at this set of ideas from a symplectic perspective. People have done this for projective varieties (see for instance ([11], [21], [26] and [24]) but not so much for open varieties. An affine variety is called uniruled if through every point there is a rational curve passing through that point. It is rationally connected if through every pair of generic points, there is a rational curve passing through these points.

Theorem 4 If $\mathrm{A}$ and $\mathrm{B}$ are symplectomorphic affine varieties then $\mathrm{A}$ is uniruled if and only if $\mathrm{B}$ is uniruled.

A topic for further research would be to ask the same question for rational connectedness.

Kollar ([12]) showed that the fundamental group of rationally connected open varieties is governed by the fundamental group of the respective ra- tional curves. There is a notion of rational connectedness for symplectic manifolds defined in terms of Gromov Witten invariants. If we have an open subset of a rationally connected symplectic manifold given by the comple- ment of some nicely intersecting symplectic submanifolds then most of the 
fundamental group of this open set should be generated by the fundamental group of a rational curve. In particular rationally connected compact sym- plectic manifolds should have trivial fundamental group. This work will be used to show that low degree symplectic hypersurfaces in complex projective space whose dimension is greater than 2 are simply connected.

An Exotic Stein manifold is a Stein manifold diffeomorphic but not sym- plectomorphic to $\mathbf{C}^{\mathbf{n}}$. Much of my research in this past has been related to the study of exotic Stein manifolds. This research in turn has led to further research by others (see [13], [3, Chapter 11]). Every Stein manifold has a special handle decomposition which is called a Weinstein handle decompo- sition. This is a very good way of describing Stein manifolds symplectically. A Stein manifold has infinitely many different such decompositions. We say that it is of finite type if it admits a Weinstein handle decomposition with only finitely many handles. Given some finite Weinstein handle decomposition, we have a contact manifold called its contact boundary. In this field we can ask the following questions:

(1) Do there exist exotic Stein manifolds? Can these be smooth affine varieties?

(2) How many of them are there?

(3) More generally given a Stein manifold M, how many others can we find that are diffeomorphic but not symplectomorphic to $\mathrm{M}$ ?

(4) If these exotic Stein manfiolds are of finite type, then can we show that their contact boundaries are different as contact manifolds?

(5) Can we find non-finite type Stein manifolds that are diffeomorphic but not symplectomorphic to finite type Stein manifolds? How many of them are there?

(6) Given two exotic Stein manifolds with an explicit Weinstein handle decomposition is there some algorithm telling us if two are the same or not.

The first example of an exotic Stein manifold was constructed in [22]. There are many examples of Stein manifolds that are non-finite type for purely

topological reasons. For instance we can use the infinite genus surface $\left\{y^{2}=\sin (x)\right\}$. We show that such manifolds exist even if they have finite topology.

Theorem 5 (unpublished, but described in [23, Section 7] and also proven in [15, Theorem 1.1]). There is an exotic Stein manifold in every complex dimension greater than 2 such that every Weinstein handle decomposition has infinitely many handles. This is the first example of a Stein manifold that is diffeomorphic but not symplectomorphic to a smooth affine variety because affine varieties are of finite type.
In fact exactly the same techniques can be used to show that for every Stein manifold $\mathrm{M}$ of complex dimension greater than 2, there is another non-finite type Stein manifold $\mathrm{M}^{0}$ diffeomorphic to $\mathrm{M}$. We use a symplectic invariant called symplectic homology to prove this result. We can ask if there are exotic finite type Stein manifolds.

Theorem 6 [16]. There are infinitely many pairwise nonsymplectomorphic exotic finite type Stein manifolds in each dimension greater than 2 .

These examples are built from standard examples in algebraic geometry. We show that symplectic homology as an algebra has a different number of idempotents for each of our exotic finite type Stein manifolds. A current project is to improve the above theorems as follows:

Theorem 7 For all $n \geq 4$, there are infinitely many smooth affine varieties diffeomorphic to $\mathrm{C}^{\mathrm{n}}$ that are pairwise nonsymplectomorphic. For any finite type Stein manifold $M$ there are uncountably many non-finite type Stein manifolds diffeomorphic to $\mathrm{M}$ that are pairwise non-symplectomorphic.

The smooth affine varieties are standard examples constructed using a method called the Kaliman modification. Again we use the algebra structure of symplectic homology to distinguish them. Each smooth affine variety in the previous theorem has a contact boundary which is diffeomorphic to the sphere. One can ask if we can distinguish these boundaries. I hope to prove the following claim:

Claim 3 Let $X$ be a smooth affine variety. Then there is a finite list of algebras $A_{1}, \cdots, A_{k}$ such for any other Stein manifold $\mathrm{S}$ diffeomorphic to $\mathrm{X}$ where this diffeomorphism is a symplectomorphism outside a compact set, $\mathrm{SH}_{*}(\mathrm{~S})=$

$\mathrm{A}_{\mathrm{i}}$ for some i. Symplectic homology here has to have coefficients in a finite field.

If we combine the above claim with Theorem 7 then we get the following corollary:

Corollary 8 There are infinitely many contact structures on $\mathrm{S}^{2 \mathrm{k}+1}$ for $\mathrm{k} \geq 3$. These are the contact boundaries of exotic Stein manifolds.

This is an improvement of a theorem by Ustilovsky in [25] which shows that there infinitely many contact structures on $\mathrm{S}^{4 \mathrm{k}+1}$ for $\mathrm{k} \geq 1$.

If one is given an explicit presentation of two Stein manifolds then it is natural to ask if there is some recipe which tells us if they are symplecto- morphic or not. It turns out that this is impossible. For instance one can use 
Weinstein handle attaching to construct for each group presentation $\mathrm{P}$ an explicit Stein manifold MP whose fundamental group is GP and such that if the associated group $\mathrm{GP}_{\mathrm{P}}$ is trivial then $\mathrm{MP}_{\mathrm{P}}$ is symplectomorphic to $\mathrm{M}_{\mathrm{h}} \mathrm{i}$ where hli is the empty presentation. The above problem is undecidable because if we could solve it then we could also solve the group word problem which is impossible. This undecidability problem is true for topological reasons. We can show that it is true for purely symplectic reasons:

Theorem 9 [14]. For every group presentation $\mathrm{P}$ we can construct an exotic Stein manifold MP of complex dimension greater than 7 with the property that MP is symplectomorphic to $\mathrm{M}_{\mathrm{h} \mid \mathrm{i}}$ if and only if the associated group GP is trivial. A similar result holds for contact structures on the odd dimensional sphere of dimension greater than 13 .

A tool called the growth rate of symplectic homology is used here to prove our result.

In this field of research we are interested in the following questions:

(1) Which symplectomorphisms of Stein manifolds have fixed points?

(2) If I have a contact boundary of a Stein manifold then does every supporting contact form have a Reeb orbit? This is related to a conjecture called the Weinstein conjecture.

(3) Let p be a point on a Stein manifold and suppose we have two Hamiltonians $\mathrm{H}$ and $\mathrm{K}$. Will the flowlines of $\mathrm{H}$ and $\mathrm{K}$ passing through $\mathrm{p}$ ever intersect again? If they do intersect again and $\mathrm{K}$ flows for time 1 then $\mathrm{p}$ is called a leafwise intersection point.

Theorem 10 [2]. We construct many Hamiltonians $\mathrm{H}$ whose domain is a Stein manifold along with a chosen energy level of $\mathrm{H}$ such that for any generic Hamiltonian $\mathrm{K}$ there are infinitely many leafwise intersection points lying on this energy level.

Many Stein manifolds admit special Weinstein handle decompositions called Lefschetz fibrations. We start with the contact boundary of the Stein manifold obtained by cutting out a smooth fiber from a Lefschetz fibration with at least on singularity. Let $\mathrm{S}$ be any Stein manifold with the above con- tact boundary $\mathrm{C}$ as a submanifold. Then our Hamiltonians $\mathrm{H}$ in the above theorem are ones which have $\mathrm{C}$ as a level set and whose flow on this level set is the Reeb flow. In order to show that there are many leafwise intersection points for any generic Hamiltonian $\mathrm{K}$ we use group called Rabinowicz Floer homology. We have similar dynamical results for many Hamiltonians on affine varieties with positive log Kodaira dimension.
Let $\mathrm{Q}$ be a simply connected manifold such that the Betti numbers of the free loopspace $\mathrm{Q}^{1}$ are unbounded. For instance this property holds if the cohomology algebra of $\mathrm{Q}$ has at least two generators. A celebrated result by Gromoll and Meyer [9] says that Q has infinitely many closed geodesics that are not multiple covers of other closed geodesics. This theorem is not trivial due to the fact that these closed geodesics can be very degenerate.

We can generalize the above result as follows: The unit cotangent bundle $\mathrm{S}^{*} \mathrm{Q}$ is the set of covectors of length 1 . This has a natural contact form and its Reeb flow is the geodesic flow.

Theorem 11 [17] Any compatible contact form on $S^{*} Q$ has infinitely many Reeb orbits.

We use an invariant called local Floer homology here to prove this com- bined with results from [8]. Using the above techniques we can also prove the following theorem:

Theorem 12 [17]. Let $\mathrm{C}$ be the contact boundary of some Stein domain of complex dimension greater than 3. Then there exists a (possibly different) contact manifold $\mathrm{C}^{0}$ diffeomorphic to $\mathrm{C}$ with the property that every supporting contact form has infinitely many Reeb orbits.

Using an invariant called persistent symplectic homology it might be pos- sible to give examples of many manifolds where the rate at which the number of Reeb orbits grows with respect to length is logarithmic. This is a future research direction.

Every Lefschetz fibration on a Stein manifold $M$ has a monodromy map $\varphi: F \rightarrow F$. To such a symplectomorphism $\varphi$ we can associate a Floer homology group $\mathrm{HF}_{*}(\varphi)$. The chain complex for this homology group consists of fixed points of $\varphi$. We have the following result (roughly):

Theorem 13 [18] (Accepted for publication in Selecta Mathematica.) There is a spectral sequence with $\mathrm{E}^{1}$ page given by

$\mathrm{HF}(\mathrm{M}, \varphi): \pm \mathrm{H}^{\mathbf{n}-*}(\mathrm{M}) \oplus^{\mathbf{M}} \mathrm{HF}\left(\varphi^{\mathrm{k}}\right)$

converging to symplectic homology.

$\mathrm{i} \geq 1$ 
As an application of this spectral sequence we show that a certain infinite family of compactly supported symplectomorphisms $\varphi$ have a fixed point.

Suppose we have a compact Lagrangian L inside a Stein manifold. A small neighbourhood of this Lagrangian is symplectomorphic to a small neighbourhood of the zero

section of its cotangent bundle $\mathrm{T}^{*} \mathrm{~L}$. One question is, how big can this neighbourhood be? I hope to use tools such as symplectic homology and wrapped Floer homology to address this question. The following is joint work with Strom Borman.

One family of Lagrangians called exact Lagrangians have arbitrarily Large such neighbourhoods. The aim of this project is to find other Lagrangians which cannot have too big neighbourhoods. For some Lagrangian $\mathrm{L}$ we can define $\mathrm{c}(\mathrm{L})$ to be the largest $\mathbf{r}$ so that $\mathrm{B}\left({ }^{\mathbf{P}} \underline{\mathbf{r}}\right)$ symplectically embeds into our Stein manifold and so that the

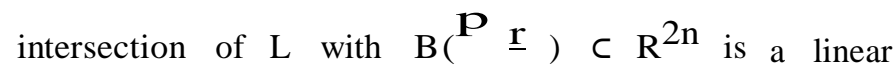
Lagrangian passing through 0 . We can also define another number $e(L)$ which is called the displacement energy. This basically measures the size of the smallest Hamiltonian needed to displace $\mathrm{L}$ from itself.

Claim 4 If $\mathrm{L}$ admits a metric of non-positive curvature then then $c(L) \leq 4 e(L)$ where $e(L)$ is the displacement energy. In particular $\mathrm{c}(\mathrm{L})$ is finite for any such Lagrangian inside $\mathrm{R}^{2 \mathrm{n}}$.

The proof of this claim involves calculating wrapped FLoer homology of a non-compact exact Lagrangian which intersects L in a particular way. It would be interesting to see if we can relax the curvature constraint on L.

\section{REFERENCES}

[1]. Alberto Abbondandolo, Matthias Schwarz, and Alessandro Portaluri, The homology of path spaces and floer homology with conormal boundary conditions, Journal of Fixed Point Theory 4 (2008), no. 2, 263-293.

[2]. Peter Albers and Mark McLean, Non-displaceable contact embeddings and in- finitely many leaf-wise intersections, J. Symplectic Geom. 9 (2011), 271-284, arXiv:0904.3564.

[3]. K. Cieliebak and Y. Eliashberg, Symplectic geometry of Stein manifolds, In prepara- tion.

[4]. Yakov Eliashberg, Topological characterization of Stein manifolds of dimension > 2, Internat. J. Math. 1 (1990), no. 1, 29-46.

[5]. Symplectic geometry of plurisubharmonic functions, Gauge theory and sym- plectic geometry (Montreal, PQ, 1995), NATO Adv. Sci. Inst. Ser. C Math. Phys. Sci., vol.
488, Kluwer Acad. Publ., Dordrecht, 1997, With notes by Miguel Abreu, pp. 49-67.

[6]. Yakov Eliashberg and Mikhael Gromov, Convex symplectic manifolds, Several com- plex variables and complex geometry, Part 2 (Santa Cruz, CA, 1989), Proc. Sympos. Pure Math., vol. 52, Amer. Math. Soc., Providence, RI, 1991, pp. 135-162.

[7]. Robert Friedman and John W. Morgan, Algebraic surfaces and Seiberg-Witten in- variants, J. Algebraic Geom. 6 (1997), no. 3, 445-479.

[8]. Ginzburg, V. L. and G "urel, Z., Local Floer Homology and the Action Gap, (2008),

1-28, arXiv:SG/0709.4077v2.

[9]._Detlef Gromoll and Wolfgang Meyer, Periodic geodesics on compact $\pi$ riemannian man- ifolds, J. Differential Geometry 3 (1969), 493-510.

[10]. Mark Gross, Paul Hacking, and Sean Keel, Mirror symmetry for log calabi-yau sur- faces i, arXiv: 1109.4466. 